\title{
LET THE STUDENTS DESIGN THEIR OWN EXPERIMENTS!
}

\author{
Karen A. Lawrence \\ W. Booth School of Engineering Practice \& Technology, McMaster University \\ lawrek@mcmaster.ca
}

\begin{abstract}
The objective of this paper is to illustrate the use and benefits of a student-directed Design of Experiments (DOE) project as an active learning instrument within a second course in statistics for students enrolled in one of three programs in the Bachelor of Technology at the W. Booth School of Engineering Practice and Technology, McMaster University. Pedagogy will be considered, learning outcomes presented, level and depth of topic areas will be explored and evidence of benefit to the students will be shared. Examples of student objective statements will be given to show the level of interest in conducting a self-chosen experiment. Concluding comments from student reports will be highlighted to demonstrate how the project serves as a useful vehicle for discussing practicalities that arise in real life investigations. Lastly, details about the yearly American Society for Quality (ASQ) Student Quality Showcase event will be shared to demonstrate how interaction with industry professionals enhances student confidence and develops key attributes desired in engineering professionals.
\end{abstract}

Keywords: engineering education, active learning, statistics education, design of experiments, student projects, pedagogy, industry, engineering graduate attributes

\section{INTRODUCTION}

The Canadian Engineering Accreditation Board (CEAB) identifies twelve graduate attributes which include general life competencies such as problem analysis, investigation, teamwork and communication skills. Arguably, these attributes align with the competencies and character qualities identified by the World Economic Forum as skills required for the $21^{\text {st }}$ century. [12]

Active learning has received considerable attention in recent years, and there is strong support for all forms to increase student engagement [9]. Active learning in the form of group projects can capitalise on student engagement and develop the required general life competencies. Students completing projects of his or her devising, from start to finish, gain considerable experience regarding practical questions that naturally emerge and learn to confront real problems which do not merely mimic the ones that are academically created for a class. [6]
Statistical education for engineers at universities is generally inadequate, delivered as one course and tends to focus on theory and more mathematical aspects rather than the techniques which are more practically useful to the engineering profession [2]. Statistical techniques and the ability to interpret results are critical components of problem analysis and investigation - attributes desirable for accreditation by CEAB. Engineering students need to get beyond calculations and prescribed formulas; they benefit from statistical education beyond the typical first course. Follow on courses should be geared towards the type of statistical work most engineers face in their careers. Besides, engineering students need to see statistics not as an imposition, but as an exciting learning experience to be applied in their professions [3].

How does an engineering program help students develop problem analysis and investigation skills, fill the cognitive gap in statistics, engage them in their learning and include an element of fun...all at the same time? First, offer a second course in statistical methods that covers design of experiments (DOE). Second, include in the course a project where students plan, design, perform, analyze and report on a topic/problem of their choice. Lastly, give students a productive and fulfilling opportunity to share their projects with industry professionals.

\section{THE LEARNING LANDSCAPE}

\subsection{The Program}

The Bachelor of Technology program (B.Tech) is a skills-based engineering technology and management program at the university level. The program is a partnership between McMaster University's W. Booth School of Engineering Practice and Technology (SEPT) and Mohawk College's School of Engineering Technology. The program combines traditional lectures with hands-on practice in modern high-tech labs, along with real-world industry experience obtained through 12-months of workplace co-op in one of three areas of program specialization: Automotive and Vehicle Engineering Technology, Biotechnology, and Automation Engineering. The B.Tech program uniquely blends engineering technology with management and societal courses to explore the human side of engineering. The outcome is T-shaped graduates equally savvy in technical as well as management skills, and 
who have the breadth of perspective to tackle broadbased engineering issues in creative and practical ways.

\subsection{The Course}

All students regardless of specialization complete a first course in Engineering Statistics as well as a second course that connects statistics, industry and technology through the lens of quality improvement. This second course - Quality Control and Assurance Methods focuses on the planning, measurement, control, and improvement of quality using statistical tools, tests, and methods such as Design of Experiments (DOE), Taguchi methods, control charts, capability, and measurement systems analysis. Quality Management strategies (i.e., Six Sigma) and ISO standards are introduced. Course assessment includes software laboratory tutorials, two student-directed projects, a midterm and a comprehensive final examination.

The course has five learning outcomes, two of which are dedicated to the DOE project*:

1. Demonstrate an understanding of Quality Management Strategies and ISO 9000 standards and their complementary function in operations.

2. Select appropriate statistical process control tools to determine if a process is running within acceptable industrial standards with the aid of statistical software.

3. Integrate statistical process control techniques within a framework of quality improvement.

4. *Design statistical experiments and verify the benefits and limitations of different types of designs (including Taguchi techniques) with the aid of statistical software.

5. Measure gage capability and determine the components of variation in a measurement process.

6. *Plan, design, perform, analyze and report on a statistically designed experiment with the aid of statistical software.

Approximately four weeks at the beginning of the term are dedicated to the topic of DOE. DOE is defined as a branch of applied statistics that deals with planning, conducting, analyzing, and interpreting controlled tests to evaluate the factors that control the value of a parameter or group of parameters [1]. Coverage includes a review of statistical methods emphasizing the importance of graphical data representations, randomization, variability, the multivariate nature of physical systems, analysis of variance and regression modeling. The coverage continues with the design elements and practical considerations of 2-level factorial experiments and fractional factorials. One week of content is dedicated to the calculation of effects, model diagnostics, and analysis procedures. Concepts such as randomized runs, blocking, replications, error, interactions, and confounding are carefully woven through the content to ensure students are critically assessing the results generated. Competency in statistical software to create the design matrix as well as to produce graphs and diagnostics required for analysis is developed through highly prescribed and detailed tutorials.

\subsection{The DOE Project Description}

The DOE project is a group effort and consists of four components:

Proposal: Decide on an experiment with three or more factors (2 levels). Experiments to consider can be of the home type or laboratory. Advice and a list of previous experiments from B.Tech students (as well as a list from another author) are available. Consultation with the instructor is essential and approval from lab coordinators required, if applicable. Proper design concepts must be considered including replication, randomization, blocking, centre points, constraints, aliasing, and choice of response(s).

Experiment: Conduct the experiment based on the project proposal as soon as possible in case some of the levels chosen are inappropriate.

Analysis: The data should be analyzed using the appropriate software (Minitab). This analysis must include ANOVA tables, effects plots (contours, if required), residual diagnostics, model selection, optimum settings and prediction. Confirmation runs are always a good idea if time/resources permit.

Report or Poster: The report/poster will be a summary of the design, the experiment and the analysis. Interpretation and conclusion are critical. Put the details of the analysis (output) in an appendix.

The project is assigned after the second lesson in DOE. The students are given one week to submit a proposal and three weeks to complete the experiment and submit a report or poster.

\subsection{Sharing with industry}

Every year since 2014, SEPT welcomes the Hamilton Section of the American Society for Quality (ASQ) to McMaster University for the ASQ Student Quality Showcase [5]. The purpose of the Student Quality Showcase Event is to bring together SEPT faculty, staff, students, and the American Society of 
Quality (ASQ) Hamilton Section to learn how quality tools, ideas, and expertise can be used to impact enterprises positively. The event provides a platform for B.Tech students to present their DOE project and to compete for monetary awards graciously donated by the ASQ Hamilton Section. The event is also an opportunity for students to meet with quality professionals from a variety of industries in the Hamilton area. Members from the ASQ Hamilton Section judge the event.

Any group who completes a DOE project is eligible to submit a poster for the ASQ Student Quality Showcase event. On the evening of the event, groups are expected to be with their poster and field questions from judges. Monetary prizes for first, second and third place winners are given. The event takes approximately two hours and provides lively interactions between students and professionals with passion on both sides. The event provides a unique opportunity to have students engage outside of an academic measure (no grade and instructors do not judge) and to talk to professionals outside the most common interaction - a job interview. The extra work required by students in a hectic term is recognized by awarding a $2 \%$ bonus for participation. Students are encouraged to think longterm and to put the completed poster in digital form on a personal website or LinkedIn profile as a means to differentiate themselves in the labour market for future employers. The evening is a community and industry event great for networking with professionals who are either directly in the quality field or who hold a peripheral interest in the role quality tools play in industry.

\section{DISCUSSION}

Topics for the project are left completely open and up to the students with some restrictions regarding legal substances and safety. Students are encouraged to pick something they care about and for which they have access to materials. Students in the Automotive program stream are encouraged to coordinate the DOE project with a lab requirement from their Manufacturing course. The lab requires students to design, machine, 3-D print and mill a moving part/mechanism. One of the best projects to come from this coordinated effort is a student-built trebuchet that was optimised using DOE methods. The better the connection the students have to the project topic, the better the experience with the assignment.

DOE content (specifically factorial experiments) is introduced with the brownie experiment. The brownie experiment is a 3-hour lesson plan that walks students through the complete procedure for a designed experiment using the semi-hypothetical problem of baking the best tasting brownies. Students are asked to brainstorm response variables and factors that affect the response variables. One response is picked (e.g., taste) and proper measurement of the response is carefully considered. Once the response measurement system is clearly defined, a cause and effect diagram (Ishikawa diagram) is introduced and factors affecting taste are collected. The lesson picks up speed and by the end encompasses the handling of noise factors, design matrix development, collection of data in random run order, interpretation of effects, model building and the optimal settings for the best tasting brownie. The entire lesson is completed by hand on the board with students providing information, suggestions and doing the calculations. At the end of three hours, the students have a suite of new vocabulary words plus a rich collection of methods and necessary skills to tackle their experiments. On a secondary level, many students learn for the first time how to make brownies from scratch.

\subsection{Types of experiments}

Are there enough possibilities for experiments when students select their topics? Absolutely! Hunter [6] provides a list of some projects reported by students in his experimental design course over his very long teaching and consulting career. The list contains 101 ideas (topic, factors, and response), sixty-three of which are of the home type and thirty-eight are laboratory based. A list of projects completed by past B.Tech students includes 132 topics collected between 2014 and 2017 based on proposals submitted. Both lists are provided to students.

The breadth of project topics is best relayed through the students' own words. Online gaming is popular and offers the opportunity to compare physical systems to computer simulations:

"By observing and comparing the results of the
player's post-match rating while changing the
factors allows our group to make conclusions on
how they affect our player and if FIFA 2017 is
an accurate simulation of a real-world soccer
match."

Many students choose physical activities and match the project to one or more of the group member's abilities:

"Cycling was chosen through two main considerations, cost, and ease of running the experiment. Justin, a member of the group, has been cycling for the past couple years so is comfortable with the area, knows the roads that can be used for this experiment, and has all of 
the equipment for the experiment readily available."

Students are encouraged to explore topics in their chosen technical subject but only if the technical subject does not overshadow the objective of the project (to learn DOE). Automotive students are keen to have a go at automotive problems that affect every day driving:

"Are there any benefits of putting 91 octane in a car that has an engine designed for 87 octane? Will it be more efficient?"

"The objective of this experiment is to isolate some of these factors and see how they alter the emergency braking distance of a passenger vehicle."

Biotechnology students tend to lean towards chemical or pharmaceutical experiments:

"The experiment is being performed to test the quality of Bayer Alka-Seltzer tablets. A major topic in pharmacokinetics is the absorption rates of pharmaceutical products."

Automation Engineering students are the most diverse and these students are the ones that pick topics on our current use of digital tools:

"Battery technology has been improving every year in the mobile market."

Practical considerations of student day to day life show up every year:

"Travel time is always a big concern for the students who live off the campus."

"Typing has become one of the most essential skills required for anyone who sees himself/herself working with a computer in some capacity. So, learning to type fast has its benefits whether it is for at work in an office or in class."

Fun topics abound and students usually end up with new insights that connect their technical education to activities they enjoy:

"The purpose of this experiment was to optimize a game called 'water pong'. Often played with beer in the place of water, this game has been a pastime of many post-secondary students for decades."

\begin{abstract}
"When solving any type of puzzle, concentration \& mental ability are huge factors in completion. The objective of this experiment was to determine which distraction factor has the biggest impact on the completion time for a Rubik's cube, which in general determines the individual ability to problem solve under certain conditions."
\end{abstract}

\subsection{What do students learn?}

The project can be a transformative experience. Involvement in a study from topic inception to conclusion, from brainstorming to report writing, naturally results in a more profound understanding than typical pedagogical approaches. Students able to communicate the details of the experimental process as well as the difficulties they face. Communication is a key role of the engineer [7] and the report or poster provides written evidence of their ability to do so. Learning achievements are best relayed through the students' own words:

"Overall the experiment was a very effective way of analyzing the MCK (vibration) system properties with respect to acceleration and enabled the students to apply the principles of DOE learned in lecture and tutorial to design and carry out their own with experiment with confidence."

Students learn to carefully select response variable(s) and to manage a response that is not quantitative. Selection of the response variable is crucial as it represents the process quality characteristic to be improved or the engineering parameter to be optimized:

"The appropriate response was critical in the success of this activity. We had to ask ourselves three important questions to determine the most appropriate response, which were: what is the level of measurement, what is the importance and what is the shape?"

The importance of the response is evident in the care students give to the measurement process:

"We decided that the "distance travelled" was appropriate, and our measurement would end when the ball made first contact with the ground. To measure, we used a measuring tape and laid it down parallel to our swinging apparatus. To determine where the ball landed we would use a slow-motion camera (built into one member's iPhone), and we would run the experiment and then watch the replay back in slow-motion. We 
then chose the nearest half-inch to the ball's initial landing spot."

Some groups make crucial connections between design concepts and measurement systems for responses:

"We decided to do repetitions to increase the accuracy of our measurements but surprisingly that opened another avenue in which to analyze our results. With repetitions we could also analyze how the different settings for the dart throw can affect the precision or spread of your throws."

Choice of factors includes the consideration of factor levels (range over which the variable is experimented with). Students are encouraged to push the experimental range of a factor as far as possible within practical or feasible constraints of the system and the impact on the response. Students learn to carefully consider how to manage non-experimental factors (nuisance or background variables) and the impact these factors have on response error:

"A block was added to angle/thrust and person throwing the plane since they are considered to be nuisance variables and may cause variation within the results. The p-value for the block was $0.330[\ldots]$ therefore considered insignificant."

"First of all, the overall procedure in creating a DOE showed us that an experiment cannot yield effective data unless certain precautions are taken. It is important to block certain factors to avoid any type of nuisance error."

"Background factors such as airflow or wind, temperature, humidity and air pressure were made constant by performing the experiment inside a single room all in one day."

Simple graphical tools are an integral part of the DOE body of knowledge and are used to draw conclusions about the effect of experimental factors and diagnose model effectiveness:

"Using the interaction plot, main effects plot and box plot, the team was able to determine the optimal settings for an MCK [vibrating] systems acceleration."

"Before the model can be used to determine the optimal factors, the model assumptions using residual plots are verified. By analyzing the residual plots [...] verifies that the residuals are independent $[. .$.$] confirming that the points are$ from a normally distributed population [...] errors across all factor levels are constant."

The use of factorial experiments in DOE is the only way to ensure all process factors and their interactions are systematically considered. In many processes, interaction effects are more important than the effect of an individual factor on the response. Students learn to recognize significant interaction effects and to interpret them correctly:

"Swing length also has a significant interaction with swing height. When swing length is set to its low setting, distance increases from 11.75 to 17.625 on average as swing height changes from low to high. When swing length is set to the high setting, distance increases from 14.25 to 26.875 on average as swing height is changed from low to high."

As part of the project proposal, students are required to predict the outcomes based on existing knowledge. Such habits develop learning in the experimental process:

"After performing our experiment, we can determine that our prediction of the ping pong ball travelling the farthest when the arm length is at the high, the swing length is at the high, and the arm attachment was wedged was correct [...] the optimal settings for making the ball travel farther mostly depend on the length of the swing, [...] has the highest magnitude of effect."

Not everything goes to plan with the projects. Many design and analysis concepts become clearer when the consequences of design choices limit the students' ability to make a conclusion:

"Overall, this experiment showed us how to fully conduct a DOE and interpret the results. If we were to do this project again, we would choose more extreme conditions of each factor and shoot more balls for repeated measures."

"After completion of the experiment and becoming familiar with the DOE process a recommendation that could be made would be the inclusion of replicates into our design."

Students gain an appreciation for the efficiency of a factorial experiment and recognize DOE as the best tool to obtain valuable information about a system with minimal resource expenditure: 
"We learned how helpful $2^{\wedge} k$ factorial designs are because of the possible complexities such as $k$ being a very large value, the amount of time and money it would take to run the experiment process. But with the factorial design we can run a screening experiment with as few observations as we can to help get an idea of how the factors would affect the response variable."

\subsection{Skills, engagement and fun}

Giving the students free-reign on the project topic sets up a system where learning occurs within a creative environment. Fun naturally happens. Hunter [6] states "There is another point, and it is not the least important. Most of the students had fun with these projects. And I did, too." Students are amazed at the creative application of DOE and the tool's far reaching implications. Students are also amazed at the conclusions they reach and typically learn things that they did not know before (e.g., do not put shaving cream in a microwave oven):

\section{"The projects were both interesting and provided hands on knowledge of the course."}

"DOE project was fun because we were able to apply course material to something we enjoyed doing."

The comments from project reports given throughout this paper are evidence that the students engage with the project. Students are more comfortable and confident with DOE than they would be through testing and assignments. Many make the connection between the content learned and experiences they have had in industry:

\section{"[Instructor name] helped us understand mistakes we made in our projects and also helped us improve the project. The Projects were DOE based, something I was asked to do in my Co-op in the Aerospace industry".}

\subsection{Pedagogy}

Lecture and tutorial hours assigned to the topic of DOE and the project are spent developing a particular problem, working through the general inquiry methods and gradually surfacing the theory. There can be pressure from students to 'get on with it' and student evaluations do show that some students would prefer just to be taught content. The practical questions that arise from the student projects help to mitigate this 'spoon fed' behaviour. "Can we have missing data?" "We were thinking that oven should be a blocking variable but are we right?" "Why are none of our effects significant; what did we do wrong?" Eventually students see that the learning is in the questioning that occurs in a class and that learning is being transferred to their projects.

A prescribed template and rubric are provided to students to use for both project reports and project posters. The template (Figure 1) follows the accepted procedure for a designed experiment [8].

Planning, Conducting \& Analyzing an Experiment
1. Recognition \& statement of problem
2. Choice of factors, levels, and ranges
3. Selection of the response variable(s)
4. Choice of design
5. Conducting the experiment
6. Statistical analysis**
7. Drawing conclusions, recommendations
This is the structure of your project!II

Fig. 1. Procedure for a designed experiment

Every example in class, on the lecture slides, in the software tutorials and the textbook follow this procedure. The students gain first-hand experience with the procedure through the project. Such replication of usage develops best practices. The template makes project marking more efficient and consistent. Practitioners recognise the procedure, so student groups who participate in the ASQ Student Quality Showcase get confirmation that what they learn in the program is best practice in industry.

\section{CONCLUSIONS}

So, how does an engineering program help students develop problem analysis and investigation skills, fill the cognitive gap in statistics, engage them in their learning and include an element of fun...all at the same time? This paper has provided a clear case that the introduction of a DOE project on a topic of the students' choice can meet all these stated outcomes.

The DOE project is an active learning element in the course on quality and engages students in higher-order thinking such as planning, analysis, synthesis and evaluation. Communication and cooperation are vital skills for engineering graduates and the project provides one of many opportunities in the B.Tech program to practice these 21 st century employment necessities.

The DOE project is an opportunity to enrich engineering education in the area of statistical investigation. Although not included as a specific topic in the American Statistical Association (ASA) guidelines [4], DOE provides a platform for introducing important concepts such as randomization, efficiency, 
bias, causality, confounding and interaction. DOE also straddles the entire statistical analysis process and communication of results and through the project, students experience this with real data! The DOE project not only meets all the recommendations in the ASA guidelines for statistics education, but also bridges the education gap in engineering by providing experience in converting a process quality problem into statistical terms.

The project itself becomes an artifact for students to display and discuss as evidence of their experiential learning. Such an artifact can be useful in job applications, interviews, and professional certifications (e.g., Six Sigma). Students who choose to participate in the ASQ Student Quality Showcase develop confidence in their newly acquired abilities. As captured in an article on the 2018 event [10], one student summed up the response of his classmates, saying: "When we found out that we could keep up with the industry professionals in a technical conversation, it gave us confidence in our understanding of the methods we learned."

Students are not the only winners. Our community partners also benefit. An ASQ member states: "This showcase supports so many of ASQ Hamilton's objectives including community involvement, communication of quality principals and youth awareness. I attend these events because I enjoy meeting the students and hearing how their DOE project was planned, executed and analyzed. The principles they learn will undoubtedly benefit them in their career."[11]

Most importantly, the students have fun. Student evaluations of the course repeatedly give evidence that the best class every semester is the class with the brownie experiment. During this lesson, the room is alive with laughter while anecdotes about baking are discussed. Arguments about ingredients and preparation methods get heated while the boards gradually fill with brainstorming notations, design matrices, and effects plots. For four weeks during the winter term, conversations with students include serious debates on their projects. Course content evolves into a discussion on the best way to measure 'quality' of brewed coffee, whether fifteen factors can actually be experimented with using an online driving simulation, or whether operator (as an experimental factor) will be confounded with the choice of a barbell in a weight lifting experiment. The students joyfully discuss their experiments using new found vocabulary that has seamlessly become part of their everyday jargon. Everyone, including the instructor, benefits from this student driven, application-based method of content delivery.

For most students, it is hard to see tomorrow through the haze of a heavy academic workload. That is why face-to-face interactions with industry professionals play an essential role in career readiness. "All it takes is interest from people 'out there' for students to see themselves as part of the future," [10].

Let the students design their experiments and then let them engage with professionals in the community about their projects. Who wins? Every stakeholder in the education process wins - the student, the instructor, the program, the university, the professionals involved and the industry/society whom the students ultimately serve.

\section{Acknowledgements}

The author would like to thank the B.Tech students who continue to inspire and are always willing to take on a bit of fun. The author is indebted to Tom Sutton for his unwavering support in all things DOE. Also, I wish to acknowledge my colleague A. MacKenzie for the review of and feedback on this paper.

\section{References}

[1] American Society for Quality (ASQ) online resources https://asq.org/quality-resources/design-of-experiments

[2] Jiju Antony and Nick Capon, "Teaching Experimental Design Techniques to Industrial Engineers," International Journal of Engineering Education, vol. 14, no. 5, pp.335-343, 1998.

[3] P. Bidgoo, N. Hunt, and F. Joliffe, Assessment Methods in Statistical Education: An International Perspective. Chichester, UK: John Wiley \& Sons, Ltd. 2010

[4] GAISE College Report ASA Revision Committee, "Guidelines for Assessment and Instruction in Statistics Education College Report 2016," available at http://www.amstat.org/education/gaise.

[5] Hamilton Section of the American Society for Quality (ASQ) http://www.asqhamilton.com/

[6] W.G. Hunter, "Some Ideas about Teaching Design of Experiments, with $2^{5}$ Examples of Experiments conducted by Students" The American Statistician, vol. 31, no. 1, pp. 12-17, 1977.

[7] Isabel M. João and João M. Silva, "Student engagement with statistical design of experiments by active learning projects," 2nd International Conference on Higher Education Advances, HEAd'16.(pp203-210) ,Universitat Politècnica de València, València 2016

[8] Douglas C. Montgomery, Introduction to Statistical Quality Control. Hoboken, NJ: Wiley, 2013,768 pp. $\{978-1118146811\}$. 
[9] Michael Prince, "Does Active Learning Work? A Review of the Research," Journal of Engineering Education, vol. 93, no. 3, pp. 223-231, 2004.

[10] W. Booth School of Engineering Practice and Technology, Latest News, December 1, 2018 https://www.eng.mcmaster.ca/sept/news/industryprofessionals-help-b-tech-students-embrace-future
[11] W. Booth School of Engineering Practice and Technology, Latest News, November 29, 2017 https://www.eng.mcmaster.ca/sept/news/2017-asqstudent-quality-showcase-event

[12] World Economic Forum. (2015). New Vision for Education; Unlocking the Potential of Technology (pp. 2-7). Geneva: World Economic Forum. Retrieved from http://www3.weforum.org/docs/WEFUSA_NewVisionfo rEducation_Report2015.pdf 